\title{
Submicroscopic Malaria in Migrants from Sub-Saharan Africa, Spain
}

\begin{abstract}
Joaquín Pousibet-Puerto, $M^{\mathrm{a}}$ Teresa Cabezas-Fernández, Ana B. Lozano-Serrano, José Vázquez-Villegas, Manuel J. Soriano-Pérez, Isabel Cabeza-Barrera, José A. Cuenca-Gómez, Joaquín Salas-Coronas
\end{abstract}

In a screening program, we detected submicroscopic malaria in $8.9 \%$ of recent migrants to Spain from subSaharan Africa. Hemoglobinopathies and filarial infection occurred more frequently in newly arrived migrants with submicroscopic malaria than in those without. Our findings could justify systematic screening in immigrants and recent travelers from malaria-endemic areas.

$\mathrm{S}$ ubmicroscopic malaria (SMM) is defined as lowdensity Plasmodium infection detected only by molecular methods (1). SMM only occasionally causes acute disease but can infect mosquitoes and contribute to transmission (2).

In malaria-endemic countries, SMM prevalence varies widely. It is highest in areas of low transmission, where SMM represents a large proportion of the malaria reservoir (2). In regions to which malaria is not endemic, such as Europe, SMM prevalence is unknown but might account for up to one third of imported malaria cases (3). In areas such as Spain, Anopheles atroparvus mosquitoes can transmit strains of $P$. vivax (4), and SMM patients can be a reservoir for malaria reintroduction. We explored the frequency of imported SMM by PCR testing of a selected population of migrants to Spain from sub-Saharan Africa and describe the epidemiologic characteristics and main laboratory findings for SMM patients.

\section{The Study}

We conducted a retrospective observational study based on data obtained after the application of an SMM screening protocol in immigrant patients of sub-Saharan Africa origin seen at the Tropical Medicine Unit of the Poniente Hospital (El Ejido, Almeria, Spain) during October 2004-December 2016. This hospital's protocol comprised a series of complementary tests to screen for imported diseases, including chest and abdominal radiographs; blood count; liver and renal function tests; iron metabolism tests; serologic screening for syphilis, HIV, hepatitis B virus,

Author affiliation: Hospital de Poniente, El Ejido, Spain

DOI: https://doi.org/10.3201/eid2502.180717 hepatitis C virus, Strongyloides, and Schistosoma; and screening for fecal parasites, urine parasites, and blood microfilariae. Finally, the hospital tested for hemoglobinopathies using high-performance liquid chromatography.

The study population comprised patients who had lived in Europe for $<1$ year (newly arrived migrants [NAM]) or who had visited their home country (i.e., visiting friends and relatives [VFR]) within the previous year who sought care for any reason other than patent malaria and were screened for SMM using the conventional nested multiplex malaria PCR. The nested multiplex malaria PCR can identify 4 human malaria species (P. vivax, $P$. falciparum, $P$. ovale, and $P$. malariae) in 2 consecutive multiplexing amplifications. The first reaction amplifies Plasmodium DNA from blood samples. The second reaction enables identification of the infecting species of Plasmodium (5). SMM was diagnosed when a patient had a positive malaria PCR result and a negative direct microscopic examination result, either a thin or a thick smear, and a negative rapid diagnostic test (SD. BIOLINE Malaria Ag P.f/Pan test, Abbott, https:// www.alere.com). We excluded from the study patients $<14$ years of age and patients for whom no smear and/ or rapid diagnostic test for malaria was available. All SMM patients were treated according to World Health Organization guidelines (6).

We conducted 3 statistical analyses. First, we compared SMM patients with non-SMM patients. Then, within the SMM patient group, we compared those with and without filarial co-infection. We conducted a descriptive statistical analysis in which continuous variables were expressed as medians and interquartile ranges. Categorical variables were described as frequencies and percentages. We analyzed differences in continuous data between groups using nonparametric Mann-Whitney $U$ test and used the Fisher exact test or $\chi^{2}$ test, as appropriate, to compare categorical data. Finally, we conducted an explanatory multivariate logistic regression analysis to evaluate possible risk factors predicting SMM in the study population. The model used variables with $p<0.2$ in the bivariate analysis and those that were clinically relevant. Variables were excluded from the logistic regression model based on likelihood ratio test results (7). Hosmer-Lemeshow test and the area under the receiver operating characteristic curve were used to validate the model. We conducted statistical analyses using STATA software version 12 (https://www.stata.com). 
Of 2,719 sub-Saharan Africa patients seen, 370 (13,6\%) were included in the study (Table 1, https://wwwnc.cdc.gov/ EID/article/25/2/18-0717-T1.htm). SMM was diagnosed in $33(8.9 \%)$ patients, of whom 11 were VFRs and 22 were NAMs. The proportion of SMM was similar in both groups: $8.7 \%(11 / 126)$ for VFRs and 9.0\% (22/244) for NAMs ( $\mathrm{p}=$ 0.93 ). For SMM patients, time spent in Spain after leaving malaria-endemic areas was shorter for VFRs (2 months) than for NAMs $(6$ months $)(\mathrm{p}=0.001)$.

The Plasmodium species most frequently found was $P$. falciparum (26 [78.8\%] patients), followed by P. malariae (4 [12.1\%]), P. ovale (2 [6.1\%]), and 1 mixed parasitization by P. falciparum and P. malariae (1 [3.0\%]). Patients with and without SMM did not differ in baseline laboratory data, except for the presence of hemoglobinopathies, which occurred more frequently among SMM patients $(42.4 \%$ vs. $21.3 \% ; \mathrm{p}=0.01$ ).

When we analyzed other associated infections (Table 2), we found an important difference between SMM and non-SMM patients regarding filarial co-infection. Filariasis was present in up to $24.2 \%$ of SMM patients but in only $5.3 \%$ of non-SMM patients $(\mathrm{p}<0.01)$. Mansonella perstans nematodes were responsible of all filarial infections; in addition, 3 patients were infected by Loa loa eyeworms.

Among SMM patients, all filariasis was found in NAMs. These co-infected patients had higher IgE values $(1,080 \mathrm{IU} / \mathrm{mL}$ vs. $293.7 \mathrm{IU} / \mathrm{mL}$ [reference $0-100 \mathrm{IU} /$ $\mathrm{mL}$; $\mathrm{p}<0.01)$ and higher total eosinophil counts $(601.5$ cells $/ \mu \mathrm{L}$ vs. 270 cells $/ \mu \mathrm{L}$ [reference $20-450$ cells $/ \mu \mathrm{L}$ ]; $\mathrm{p}=0.01)$ than those who had only SMM. The coinfected group also tended to have higher platelet levels (Figure 1).
Multivariate regression analysis applied to all 370 patients showed that having filarial infection increased the odds of having SMM by 6.49 and the existence of $\geq 1$ hemoglobinopathies increased the odds by 3.93. Time after leaving a malaria-endemic area correlated inversely with risk for SMM ( $p=0.038)$ (Figure 2). For NAMs, filariasis increased the risk for SMM by 8.47 and hemoglobinopathies by 4.70 . For VFRs, however, the only risk factor was time since last visit to their home country (the shorter the time, the higher the risk).

\section{Conclusions}

Screening for SMM in patients from sub-Saharan Africa in a reference unit in Spain showed a prevalence of $8.9 \%$. The presence of filarial infection or hemoglobinopathies and a shorter time since leaving malaria-endemic areas were associated with a higher risk for SMM.

SMM is usually asymptomatic. Infrequently, it produces acute disease, especially in children (8). During pregnancy, SMM has been linked to maternal anemia and to low birth weight (9). SMM screening in risk groups, such as pregnant women and immunosuppressed persons (10), could therefore be of special interest.

Our study highlights 2 important differences between patients with and without SMM. First, the proportion of patients infected by filariasis was higher among SMM patients. In areas to which malaria is not endemic, RamírezOlivencia et al. also reported a greater number of filariasis among SMM patients than among patients with patent microscopic malaria (3). Nematodes can alter immune system response to concomitant infections, such as Plasmodium spp. Modulation of immune response produced

\begin{tabular}{|c|c|c|c|c|}
\hline Co-infection & All, no. (\%), N = 370 & Non-SMM, no. (\%), n= 337 & SMM, no. (\%), $\mathrm{n}=33$ & $p$ value \\
\hline Blastocystis hominis & $91(24.6)$ & $85(25.2)$ & $6(18.2)$ & 0.33 \\
\hline Entamoeba hystolitica/dispar & $56(15.1)$ & $53(15.7)$ & $3(9.1)$ & 0.28 \\
\hline Giardia lamblia & $25(6.8)$ & $24(7.1)$ & $1(3.0)$ & 0.35 \\
\hline Strongyloides stercoralis & 73 (19.7) & 67 (19.9) & $6(18.2)$ & 0.75 \\
\hline Hookworms & 39 (10.5) & $37(11.0)$ & $2(6.1)$ & 0.36 \\
\hline Trichuris trichiura & $11(3.0)$ & $10(3.0)$ & $1(3.0)$ & 0.99 \\
\hline Ascaris lumbricoides & $10(2.7)$ & $8(2.4)$ & $2(6.1)$ & 0.23 \\
\hline Schistosomiasis & $34(9.2)$ & $31(9.2)$ & $3(9.1)$ & 0.94 \\
\hline S. haematobium & $21(5.7)$ & $19(5.6)$ & $2(6.1)$ & 0.95 \\
\hline S. mansoni & $7(1.9)$ & $7(2.1)$ & 0 & 0.40 \\
\hline S. intercalatum & $1(0.3)$ & $1(0.3)$ & 0 & 1 \\
\hline Schistosoma spp. & $5(1.4)$ & $3(0.9)$ & $1(3.0)$ & 0.45 \\
\hline Hymenolepis nana & $7(1.9)$ & $6(1.8)$ & $1(3.0)$ & 0.64 \\
\hline Taenia spp. & $2(0.5)$ & $2(0.6)$ & 0 & 1 \\
\hline Filariae† & $26(7.0)$ & $18(5.3)$ & $8(24.2)$ & $<0.01$ \\
\hline Mansonella perstans & $26(7.0)$ & $18(5.3)$ & $8(24.2)$ & $<0.01$ \\
\hline Loa loa & $3(0.8)$ & $1(0.3)$ & $2(6.1)$ & 0.02 \\
\hline Syphilis & $39(10.5)$ & $33(9.8)$ & $6(18.2)$ & 0.12 \\
\hline Hepatitis $B$ virus & $111(30)$ & $98(29.1)$ & $13(39.4)$ & 0.17 \\
\hline Hepatitis C virus & $5(1.4)$ & $4(1.2)$ & $1(3.0)$ & 0.37 \\
\hline HIV & $2(0.5)$ & $1(0.3)$ & $1(3.0)$ & 0.17 \\
\hline
\end{tabular}

*SMM, submicroscopic malaria.

†All 3 patients infected with Loa loa eyeworms were co-infected with Mansonella perstans nematodes. 


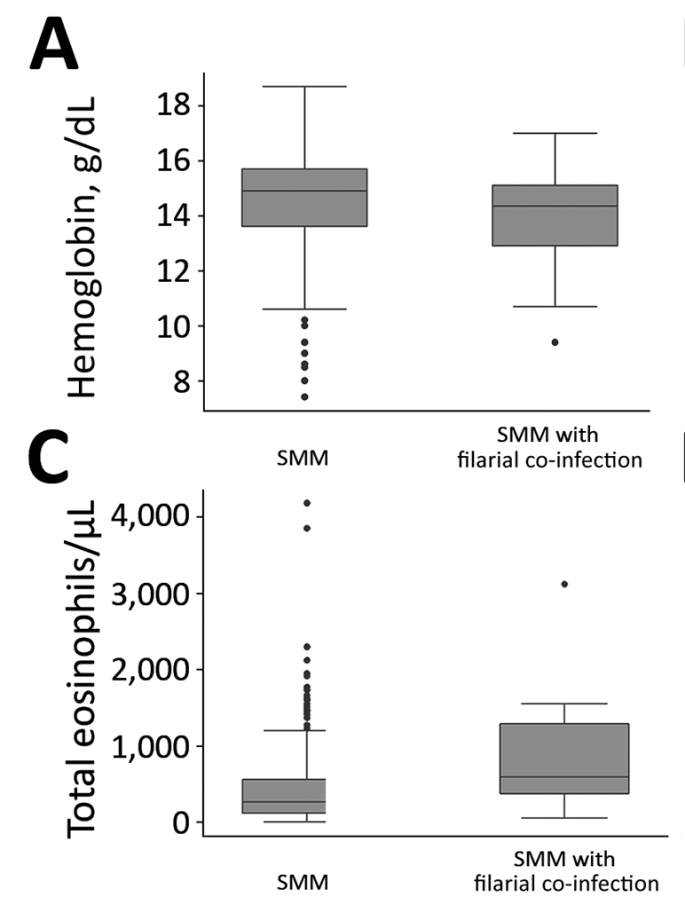

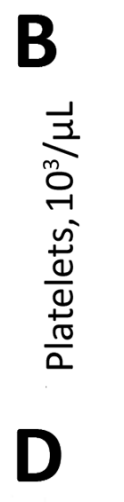
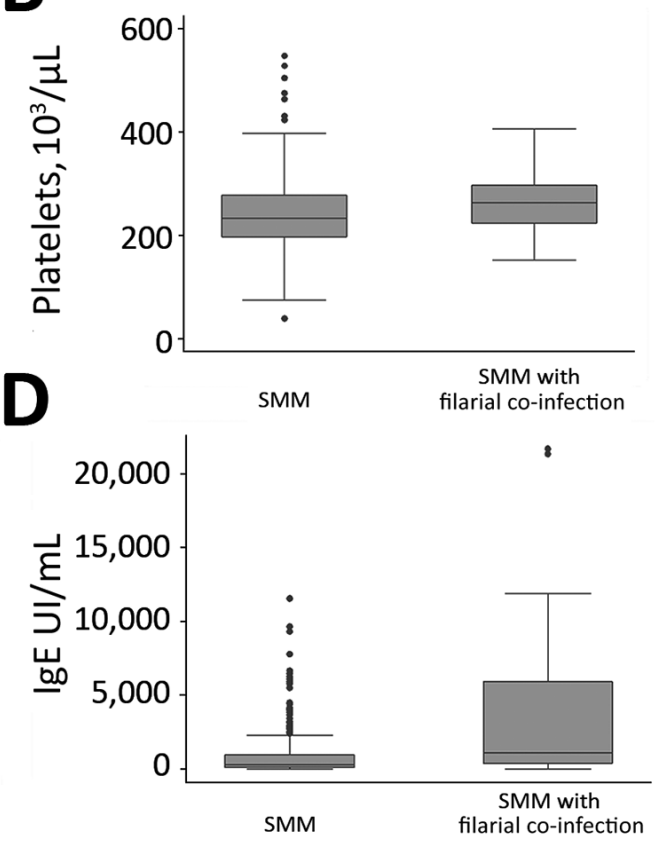

Figure 1. Differences in analytical values of blood tests among SMM patients with and without filarial co-infections, Spain, October 2004December 2016. A) Hemoglobin; B) platelets; C) total eosinophils; D) IgE. Box and whiskers plot features are defined as follows: horizontal line within box is median, bottom line of box is 25th percentile, top line of box is 75th percentile, bottom whisker is quartile $1-1.5$ interquartile range, top whisker is quartile $3+1.5$ interquartile range, and dots are outliers. SMM, submicroscopic malaria. by helminthoses, such as filariasis, might exert some protective effect against malaria, leading to lower parasitic loads, which in turn might translate into clinical protection against severe malaria $(11,12)$.

The second disparity was the presence of hemoglobinopathies, a finding much more frequent among SMM patients that resulted in an SMM risk only for NAMs.
Hemoglobinopathies exert a protective effect against severe malaria, favoring milder clinical manifestations and the existence of SMM $(13,14)$.

The relatively high prevalence of imported SMM we found could justify implementation of systematic screening in immigrants and travelers who recently stayed in malariaendemic areas, mainly for persons with risky conditions

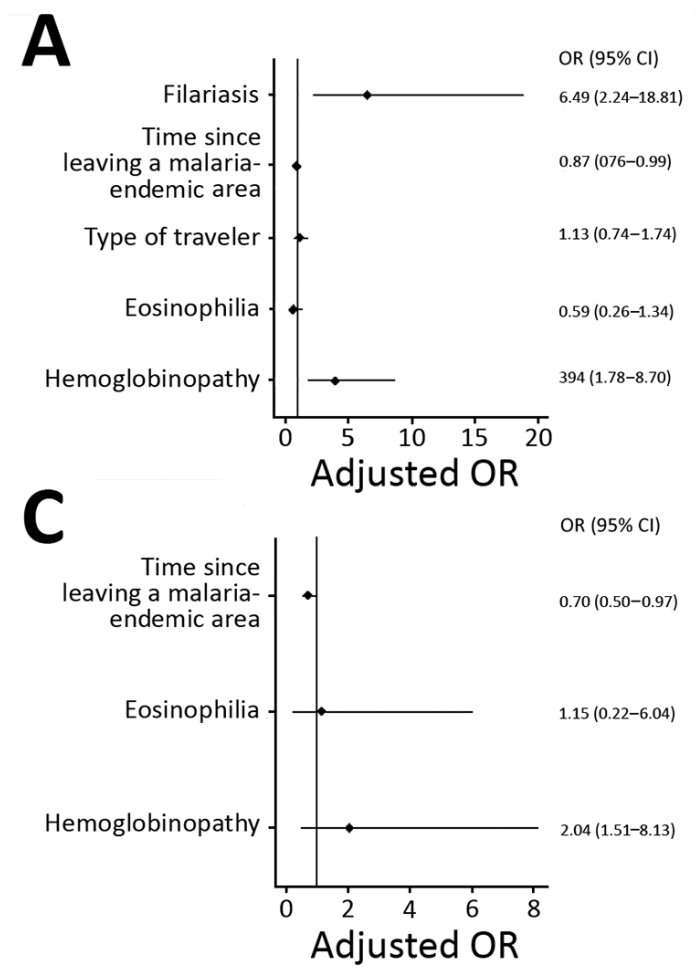

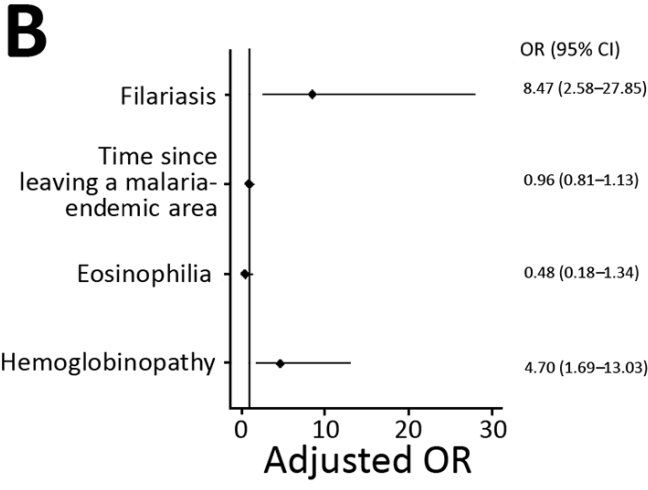

Figure 2. Multivariate logistic regression analysis for study of submicroscopic malaria in migrants from sub-Saharan Africa, Spain, October 2004December 2016. A) All patients; B) newly arrived migrants; C) migrants visiting friends and family. OR, odds ratio. 
such as immunosuppression (especially those with HIV infection) and for pregnant women. The diagnosis and treatment of SMM also can prevent future reactivations and the existence of an occult malaria reservoir in countries to which it is not endemic. Our results suggest that the presence of filariasis, hemoglobinopathies, or both should also prompt a search for SMM because these patients are at higher risk.

\section{Acknowledgments}

We thank $\mathrm{M}^{\mathrm{a}}$ del Carmen Olvera Porcel and technicians in Research Methodology and Biostatistics from the Fundación para la Investigación Biosanitaria para Andalucía Oriental Alejandro Otero for providing invaluable support for the statistical analysis of the data.

This study was conducted within the activities developed by the research group Plan Andaluz de Investigación, Desarrollo e Innovación CTS 582 of the regional Ministry of Gender, Health and Social Policy of the Government of Andalusia, RICET (Red de Investigación Cooperativa en Enfermedades Tropicales, co-financing FEDER, RD16/0027/0013) and the CEMyRI (Center for the Study of Migration and Intercultural Relations) of the University of Almeria (Spain).

\section{About the Author}

Dr. Pousibet-Puerto is a researcher of the Research Group of the Tropical Medicine Unit of the Hospital de Poniente. His primary research interests include malaria, helminthosis, and protozoal intestinal diseases.

\section{References}

1. Hermsen CC, Telgt DS, Linders EH, van de Locht LA, Eling WM, Mensink EJ, et al. Detection of Plasmodium falciparum malaria parasites in vivo by real-time quantitative PCR. Mol Biochem Parasitol. 2001;118:247-51.http://dx.doi.org/ 10.1016/S0166-6851(01)00379-6

2. Lin JT, Saunders DL, Meshnick SR. The role of submicroscopic parasitemia in malaria transmission: what is the evidence? Trends Parasitol. 2014;30:183-90.http://dx.doi.org/10.1016/ j.pt.2014.02.004

3. Ramírez-Olivencia G, Rubio JM, Rivas P, Subirats M, Herrero MD, Lago M, et al. Imported submicroscopic malaria in Madrid. Malar J. 2012;11:324.http://dx.doi.org/10.1186/1475-2875-11-324
4. Bueno-Martí R, Bernués-Bañeres A, Jiménez-Peydró R. Updated checklist and distribution maps of mosquitoes (Diptera: Culicidae) of Spain. European Mosquito Bulletin. 2012;30:91-126.

5. Rubio JM, Post RJ, van Leeuwen WM, Henry MC, Lindergard G, Hommel M. Alternative polymerase chain reaction method to identify Plasmodium species in human blood samples: the semi-nested multiplex malaria PCR (SnM-PCR). Trans R Soc Trop Med Hyg. 2002;96(Suppl 1):S199-204.http://dx.doi.org/10.1016/ S0035-9203(02)90077-5

6. World Health Organization. World malaria report 2015. Geneva: The Organization; 2015.

7. Hosmer DW, Lemeshow S. Applied logistic regression. New York: Wiley; 1989.

8. Akiyama T, Pongvongsa T, Phrommala S, Taniguchi T, Inamine Y, Takeuchi R, et al. Asymptomatic malaria, growth status, and anaemia among children in Lao People's Democratic Republic: a cross-sectional study. Malar J. 2016;15:499. http://dx.doi.org/10.1186/s12936-016-1548-3

9. Cottrell G, Moussiliou A, Luty AJF, Cot M, Fievet N, Massougbodji A, et al. Submicroscopic Plasmodium falciparum infections are associated with maternal anemia, premature births, and low birth weight. Clin Infect Dis. 2015;60:1481-8. http://dx.doi.org/10.1093/cid/civ122

10. Monge-Maillo B, Norman F, Pérez-Molina JA, Díaz-Menéndez M, Rubio JM, López-Vélez R. Plasmodium falciparum in asymptomatic immigrants from sub-Saharan Africa, Spain. Emerg Infect Dis. 2012;18:356-7.http://dx.doi.org/10.3201/ eid1801.111283

11. Metenou S, Kovacs M, Dembele B, Coulibaly YI, Klion AD, Nutman TB. Interferon regulatory factor modulation underlies the bystander suppression of malaria antigen-driven IL-12 and IFN- $\gamma$ in filaria-malaria co-infection. Eur J Immunol. 2012;42:641-50. http://dx.doi.org/10.1002/eji.201141991

12. Dolo H, Coulibaly YI, Dembele B, Konate S, Coulibaly SY, Doumbia SS, et al. Filariasis attenuates anemia and proinflammatory responses associated with clinical malaria: a matched prospective study in children and young adults. PLoS Negl Trop Dis. 2012;6:e1890.http://dx.doi.org/10.1371/journal.pntd.0001890

13. Danquah I, Ziniel P, Eggelte TA, Ehrhardt S, Mockenhaupt FP. Influence of haemoglobins $\mathrm{S}$ and $\mathrm{C}$ on predominantly asymptomatic Plasmodium infections in northern Ghana. Trans $\mathrm{R}$ Soc Trop Med Hyg. 2010;104:713-9.http://dx.doi.org/10.1016/ j.trstmh.2010.08.001

14. Crompton PD, Traore B, Kayentao K, Doumbo S, Ongoiba A, Diakite SA, et al. Sickle cell trait is associated with a delayed onset of malaria: implications for time-to-event analysis in clinical studies of malaria. J Infect Dis. 2008;198:1265-75. http://dx.doi.org/10.1086/592224

Address for correspondence: Joaquín Salas-Coronas, Hospital de Poniente, Unidad de Medicina Tropical Ctra., Almerimar sn El Ejido Almería 04700, Spain; email: joaquin.salas@ephpo.es 